\title{
A preliminary study on a patented intrauterine stent in the treatment of recurrent intrauterine adhesions with poor prognosis
}

\author{
Huan Huang, Lingxiao Zou, Aiqian Zhang, Xingping Zhao, Dabao Xu, Min Xue \\ Department of Obstetrics and Gynecology, Third Xiangya Hospital of Central South University, Changsha 410013, China \\ Contributions: (I) Conception and design: D Xu; (II) Administrative support: M Xue; (III) Provision of study materials or patients: D Xu, M Xue; (IV) \\ Collection and assembly of data: H Huan, L Zou, A Zhang; (V) Data analysis and interpretation: H Huan, X Zhao; (VI) Manuscript writing: All \\ authors; (VII) Final approval of manuscript: All authors. \\ Correspondence to: Dabao Xu, MD; Min Xue, MD. Department of Obstetrics and Gynecology, Third Xiangya Hospital of Central South University, \\ 138 Tongzipo Rd., Changsha 410013, China. Email: dabaoxu@yahoo.com; xuemin5908@sina.com.
}

\begin{abstract}
Background: Management of intrauterine adhesions (IUAs) is challenging, mainly because there is no ideal method to prevent its recurrence. Recurrence of moderate to severe IUAs after conventional treatments entails a poorer prognosis in these patients. This study aimed to assess the safety and effectiveness of a patented intrauterine stent as a barrier in the treatment of recurrent IUAs with poor prognosis.

Methods: This was a retrospective, observational study of 13 women with IUAs, admitted to the Third Xiangya Hospital of Central South University from June 2018 to September 2019. After conventional treatments, moderate to severe adhesions were still evident. Then a patented intrauterine stent was used as a barrier to prevent the reformation of adhesions after hysteroscopic adhesiolysis (HA). All cases received a second-look hysteroscopy after 2-3 menstrual cycles. American Fertility Society (AFS) scores were noted for all cases. Menstrual pattern, recurrence of adhesions, and reproductive outcomes were also evaluated.
\end{abstract}

Results: The mean number of previous HA of the 13 patients was 2.7, and mean AFS score after conventional treatments was still 8.7. Before the use of the stent, $46.2 \%$ of the patients had amenorrhea caused by IUAs. After using the intrauterine stent as a barrier, the mean AFS score was 1.7. The menstrual improvement rate was $100 \%$, while the rate of resumption of normal menstrual volume was $53.8 \%$. The second-look hysteroscopy revealed a correct position of the stents and no obvious recurrence of IUAs in all cases. After the removal of the stent, the patients were followed up for 2-13 months (mean: 7 months), and no amenorrhea was observed, the recurrence rate of IUAs was $25.0 \%$ and one patient got pregnant.

Conclusions: Even in the recurrent IUAs patients with very poor prognosis, the patented intrauterine stent has been proved to be very effective in preventing the recurrence of adhesions, on condition that its correct position inside the uterine cavity is achieved. Due to the extremely poor nature of the studied population, the recurrent rate of IUAs and pregnancy rate after the removal of the stent is still discouraging, although the improvement in menstruation is inspiring.

Keywords: Intrauterine adhesions (IUAs); adhesion reformation; intrauterine stent; hysteroscopy; hysteroscopic adhesiolysis (HA)

Submitted Dec 27, 2019. Accepted for publication Jan 14, 2020.

doi: $10.21037 /$ atm.2020.01.77

View this article at: http://dx.doi.org/10.21037/atm.2020.01.77

\section{Introduction}

With the enormous increase in elective abortion and the popularization of hysteroscopy, the incidence of intrauterine adhesions (IUAs) related to infertility and reproduction is increasing year by year (1). IUAs begin with trauma occurring to the endometrium, or infection, which results in the closure of the walls of a previously well-established intrauterine cavity causing partial or complete obstruction 


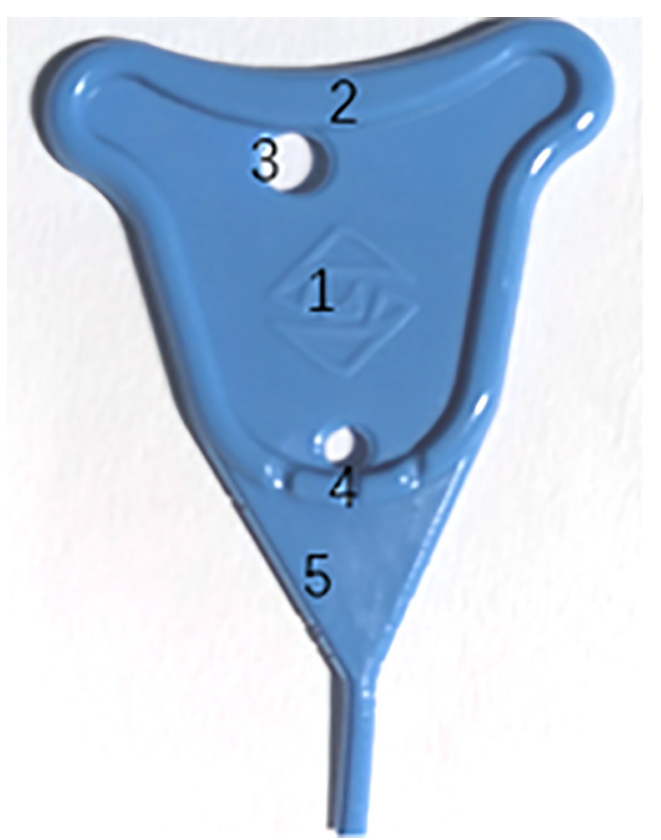

Figure 1 Illustration of the structure of the intrauterine stent. [1] A thin film about $0.5 \mathrm{~mm}$ thick in the center; [2] a stiffener about $2 \mathrm{~mm}$ thick; [3] the holes at both ends of the membrane are 2-4 mm in diameter; [4] grooves to facilitate the drainage of hematometra; [5] a triangular lower tail to prevent and treat cervical canal adhesion.

of the uterine cavity and/or cervical canal. It has been reported and studied for more than one hundred years, and is referred to as Asherman's syndrome (AS) when symptoms, such as amenorrhea, periodic lower abdominal pain, infertility, spontaneous abortion, implantation failure after assisted reproduction, recurrent abortion, and abnormal placenta develop (2-4). Usually, intervention is necessary especially for patients with characteristic symptoms and/ or fertility desire. Hysteroscopic transcervical resection of adhesion (TCRA) or hysteroscopic adhesiolysis (HA) has become the primary treatment. However, in patients with moderate or severe IUAs, the re-adhesion rate after HA has been observed to be as high as $20-62.5 \%(5,6)$. Therefore, surgical treatment combined with postoperative adjuvant therapy has become a consensus treatment of IUAs, with an added recommendation for the use of a solid or semi-solid barrier to separate the walls of the uterus for recurrence prevention (7).

For moderate and severe IUAs, HA should be performed by experienced surgeons with the aim to successfully restore the shape of the uterine cavity. The insertion of an intrauterine device (IUD), Foley balloon catheter, and hyaluronic acid gel are always applied postoperatively to prevent recurrence of adhesions $(8,9)$. After 1-3 menstrual cycles, a follow-up assessment with hysteroscopy is recommended. After receiving the aforesaid treatments, especially after more than 2 previous HA, if the followup hysteroscopy still revealed the existence of moderate to severe IUAs, these patients are clinically thought to be recurrent IUAs with poor prognosis. These patients often present with characteristic symptoms, pregnancy wastage, and infertility. Currently, there is no ideal method to prevent recurrence of IUAs, so most of those patients have to give up further fertility treatment due to lack of a better alternative. In our previous study, we developed a patented intrauterine stent (Figure 1), which had a unique design and had obtained a good clinical outcome in a randomized controlled clinical study in non-recurrent IUAs population (unpublished data). Given that those patients with recurrent IUAs have no effective means to prevent adhesions reformation, we have no alternative but to use the patented intrauterine stent. In an attempt to explore the efficacy of the patented stent for the treatment of patients experiencing poor response previously, the clinical data of 13 patients with intrauterine cavity stent application were retrospectively analyzed.

\section{Methods}

\section{Patients}

A total of 13 patients were included in the study at the Third Xiangya Hospital of Central South University from June 2018 to September 2019. All patients voluntarily participated in this study and signed the informed consent, and the study was approved by the ethics committee. The AFS scoring system of IUAs was adopted in this study (9).

The patient inclusion criteria were as follows: (I) previous hysteroscopy confirmed moderate-to-severe IUAs, and persisting moderate-to-severe IUAs after at least 1 or more conventional courses of therapy; (II) bilateral fallopian tubal ostia seen before the insertion of the patented stent during HA; (III) the size of the uterine cavity after HA matching the uterine stent; (IV) patients with fertility desire or obstruction of menstrual flow.

The patient exclusion criteria were as follows: (I) ongoing conception; (II) ovarian failure; (III) untreated genital tuberculosis; (IV) the uterine cavity being too large or too small; (V) surgical contraindications; (VI) cervical insufficiency; (VII) the intrauterine cavity still being asymmetrical after HA; 

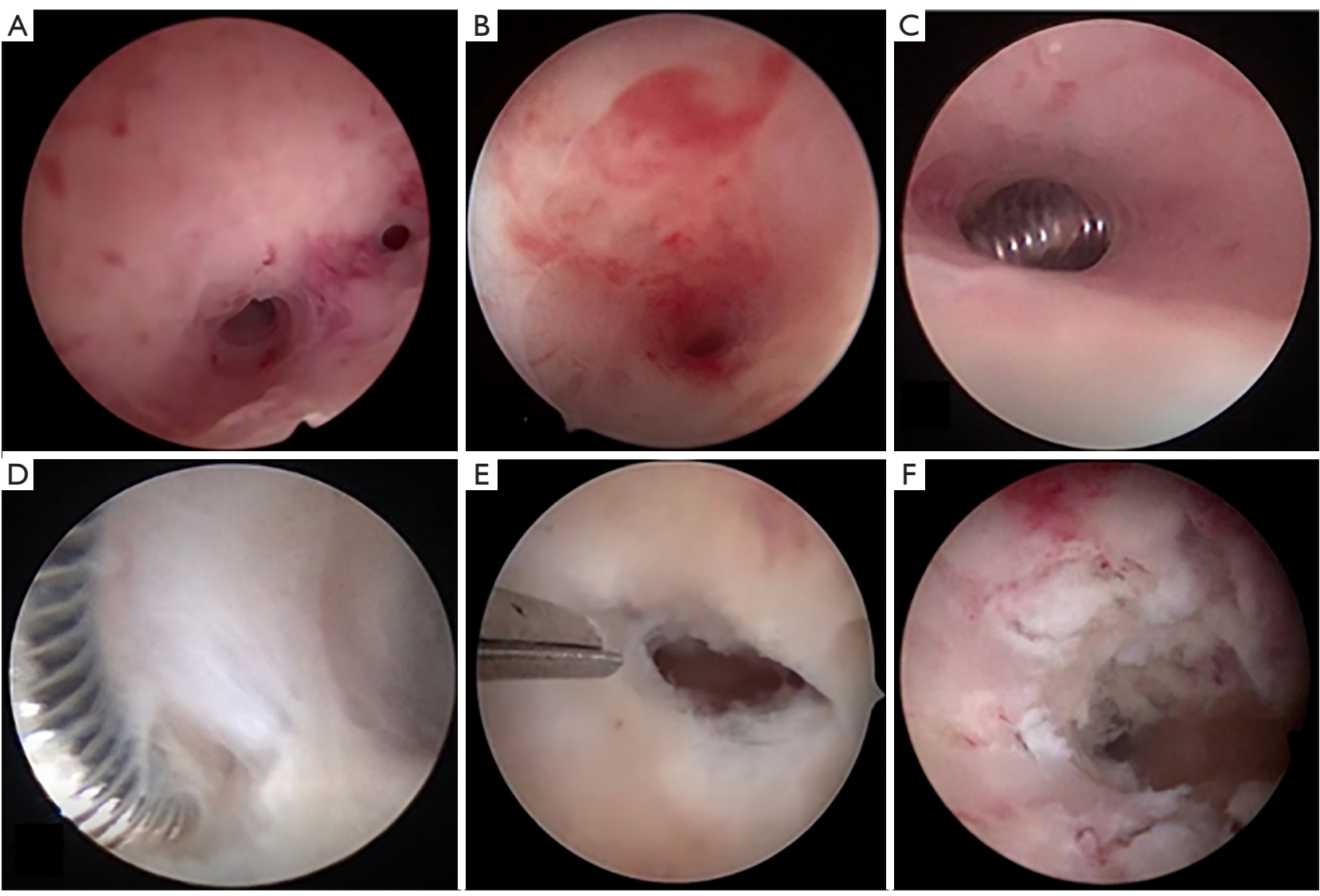

Figure 2 Hysteroscopic images of recurrent adhesions with poor therapeutic effect. (A) The adhesions of internal cervical os; (B) low segment of the uterine cavity is completely blocked by adhesions; (C) lower-middle segment of the uterine cavity; (D) an IUD surrounded by adhesions in the uterine cavity; (E) upper segment of the uterine cavity and the right uterine cornu closed; (F) the image of the uterine cavity after HA and very poor endometrium. IUD, intrauterine device; HA, hysteroscopic adhesiolysis.

(VIII) presence of malignant genital tumor.

\section{Surgical procedure}

The operation was conducted within 3-7 days after the end of the menstruation. If identified as uterine amenorrhea, surgery would be carried out at any time. Preoperative $3 \mathrm{D}$-ultrasound was performed to evaluate the size of the uterus, endometrium continuity, uterine morphology, bilateral cornua of uterine, whether the tubal ostia were visible, and the distance between the bilateral tubal ostia. The patients fasted for 8 hours before surgery, and underwent preoperative vaginal preparation and conventional sterilization. Normal saline solution was used as the distention media. Distension pressure was set at $110-120 \mathrm{mmHg}$ with a flow rate of $300-350 \mathrm{~mL} / \mathrm{min}$. All patients underwent HA after satisfactory intravenous anesthesia. The operation was performed by an experienced hysteroscopist, and monitored by transabdominal ultrasound.

The phases and steps of the procedure are detailed below.

(I) HA: a hysteroscope with a 5-Fr working channel and an outer-sheath of $5.4 \mathrm{~mm}$ in diameter was adopted to pass through the cervical canal and to determine the location and severity of the IUAs. Then, the AFS score was determined. Adhesions in the uterine cavity were separated and the scars were ploughed by using a $5 \mathrm{Fr}$ scissors (10). Alternatively, the "blunt spreading dissection technique" using double-action forceps, was carried out to dissect and retrieve the correct anatomical cavity when the internal cervical os, the lower segment of uterine cavity, or the uterine cornu was completely obstructed (11). The patients who were previously treated with the conventional therapy and prior 

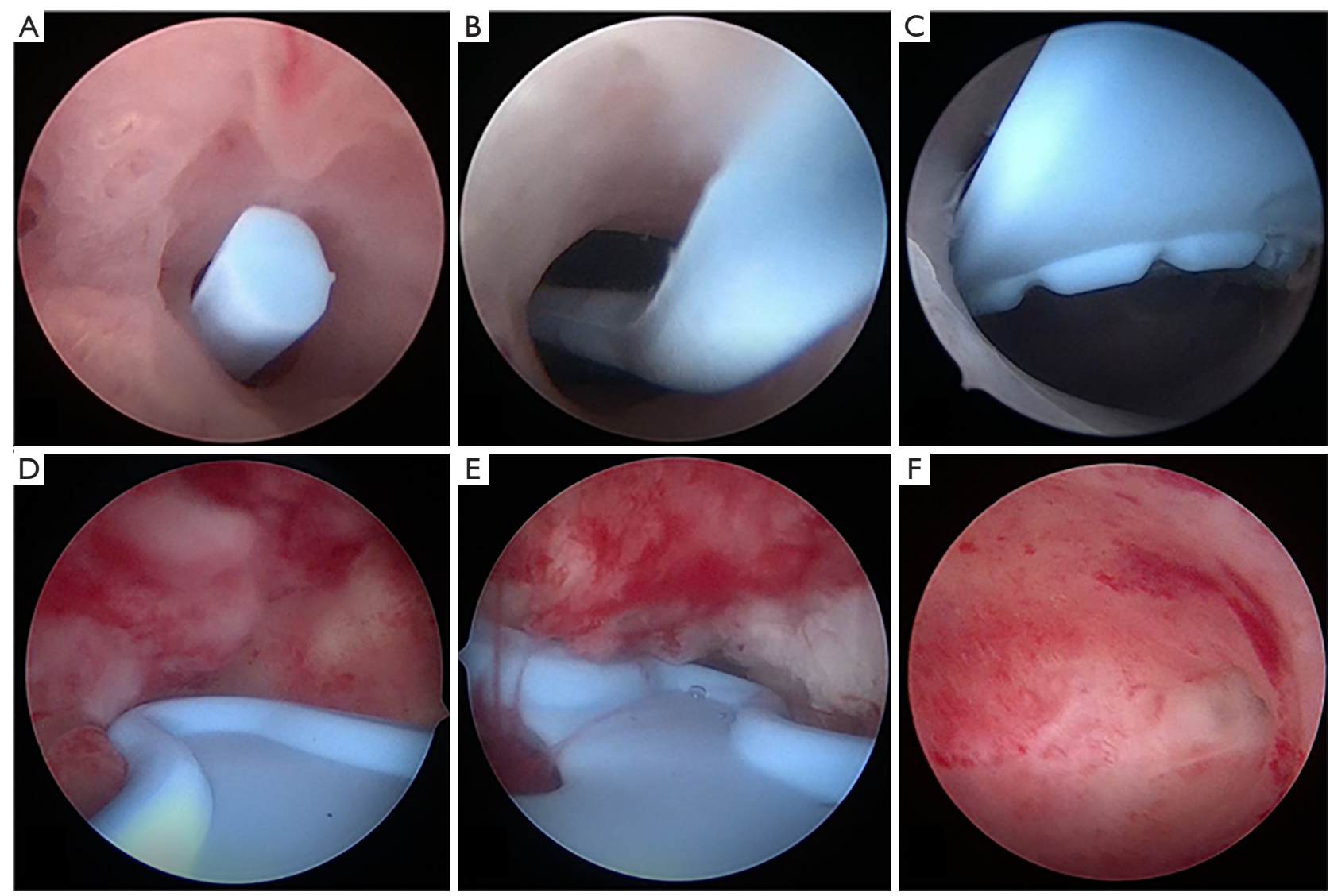

Figure 3 Hysteroscopic images of an intrauterine stent inside the uterine cavity and the normal uterine cavity. (A) The internal cervical os; (B) the lower segment of the uterine cavity; (C) the middle segment of the uterine cavity; (D) the right uterine cornu; (E) the left uterine cornu; (F) the hysteroscopic image of the uterine cavity 3 months later after HA (when the stent removed). HA, hysteroscopic adhesiolysis.

IUD insertion, were found to have dense fibrous adhesions surrounding the IUD. The latter had to be eventually removed (Figure 2).

(II) Intrauterine stent insertion: After HA, the size of the stent was determined by a combination of the hysteroscopist judgement and the predetermined intercornual distance through the preoperative 3D-ultrasound. Various sizes of intrauterine stents from XS to XL were selected depending on the size of the uterine cavity. The cervix was gradually dilated with Hegar dilators until No. 7.5, and then the stent was inserted into the uterine cavity with a pushrod. Hysteroscopy was performed immediately to confirm whether the position and size of the stent matched the uterine cavity.

(III) Postoperative management: Foley balloon catheter, hyaluronic acid gel, fresh amniotic membrane transplantation, or other auxiliary barriers were not required. If the patient had hormonal contraindications, postoperative treatment with estrogen therapy was not used; otherwise, the application of estrogen-progestin therapy after HA was considered.

(IV) Postoperative assessment: reassessment of the uterine cavity was conducted by hysteroscopy after 2-3 menstrual cycles following surgery, and the location and AFS score of IUAs were determined once again. The stent was removed at the followup hysteroscopy. All patients were advised to conceive as soon as possible after the removal of the stent. Hysteroscopic images of an intrauterine stent inside the uterine cavity during the follow-up hysteroscopy and normal uterine cavity are shown in Figure 3. 


\section{Postoperative follow-up}

Follow-up has been conducted since the insertion of the stent until present. AFS score, the recurrence of adhesions, return of menstruation or menstrual volume change, abdominal pain and pregnancy outcome were recorded.

\section{Results}

\section{Basic information before $\mathrm{H} A$ and the insertion of the stent}

The patients were aged 21-42 years old. Among these patients, trauma to a gravid uterine cavity was the factor of IUAs; $23.1 \%(3 / 13)$ had an elective abortion history, and $76.9 \%(10 / 13)$ had an adverse pregnancy among whom 5 patients had a history of missed abortion, and 5 patients terminated a pregnancy due to fetal anomaly. Furthermore, the infection rate of uterine cavity after curettage was $7.7 \%$ $(1 / 13)$, and rate of uterine artery embolization (UAE) was $23.1 \%(3 / 13)$. Also, $53.8 \%$ (7/13) of these patients appeared to have hypomenorrhea, while the other $46.2 \%(6 / 13)$ had amenorrhea. Lower abdominal pain was present in $38.5 \%$ of the patients, with 3 being in mild pain and 2 being in moderate pain. All patients had undergone multiple HA surgeries, and the mean number of previous HA was 2.7. Before they were admitted at our hospital, 46.2\% (6/13) had a history of HA in other hospitals. The initial mean AFS score at our hospital was 10.3 (ranged from 8 to 12), with a moderate degree of IUAs in $15.4 \%(2 / 13)$ and a severe degree in $84.6 \%$ (11/13) of patients. The mean AFS score after conventional treatments at our hospital was still 8.7.

\section{$H A$ and the insertion of the stent}

All HA procedures were uneventful, uterine cavities were restored to normal shape and both tubal ostia were clearly visualized. And then the stent was delivered. Among the 13 patients, 2 patients used XS size of the stent, 9 patients used $\mathrm{S}$ size and the remaining 2 patients used $\mathrm{M}$ size.

\section{After $\mathrm{HA}$ and the insertion of the stent}

There was a significant improvement in menstruation. Among these patients, 53.8\% (7/13) returned to normal menstruation, all patients who suffered amenorrhea had restored the menstruation. There was also a significant improvement in those who $(5 / 13)$ experienced lower abdominal pain, $80 \%(4 / 5)$ completely disappeared and $20 \%(1 / 5)$ experienced a relief of pain from a moderate degree to a mild degree. The follow-up hysteroscopies 2-3 months after the HA and the insertion of the stent revealed a correct position of the stents and no obvious recurrence of IUAs in all cases (13/13), but the endometrium was still extremely poor. The stents were all removed at follow-up hysteroscopies. The age, the gravidities, the presenting symptoms and AFS scores before and after the insertion of the stent were summarized in Table 1.

\section{After removal of the stent}

The patients were followed up for 2-13 months (mean: 7 months). One patient was lost to follow-up. Neither amenorrhea nor abdominal pain was observed in the remained 12 patients. And, 76.9\% (9/12) of patients experienced a gradually decreased menstrual volume and $23.1 \%(3 / 12)$ maintained normal menstrual volume. No one had lower abdominal pain. All patients were actively trying to conceive after removing the stent. Among them, one patient got pregnant, but experienced a subsequent missed abortion. And 25\% (3/12) were diagnosed as IUAs recurrence with hysteroscopy assessment. The presenting symptoms, recurrence, and outcomes of pregnancy during follow-up were summarized in Table 2.

\section{Discussion}

With damage to the endometrial basal layer, the new granulation tissue repairing the wound and dense fibrous connective tissue on the other side of the uterine cavity can fuse tissue bridges (12). IUAs are fibrotic tissues of endometrium that follow endometrial trauma or infection, resulting in partial or complete adhesions of the uterine cavity and/or cervical canal. Moreover, the large barren area of endometrium, intrauterine adhesions and highly scarred tissue may reduce menstrual volume, cause amenorrhea, lower abdominal pain, infertility, repeated abortions, and obstetric complications. In the most severe cases, the endometrial cavity can be entirely obliterated without any evidence of viable endometrium. The re-adhesion rate after HA in severe cases was reported to be as high as $20-63 \%$. Because of its high recurrence rate and potentially irreversible damage to reproductive health, IUAs have become one of the intractable problems in the field of gynecology and reproduction.

A crucial but yet unsolved question is how to most effectively maintain the cavity integrity and patency. Despite the diversity of barriers used to prevent adhesions after 


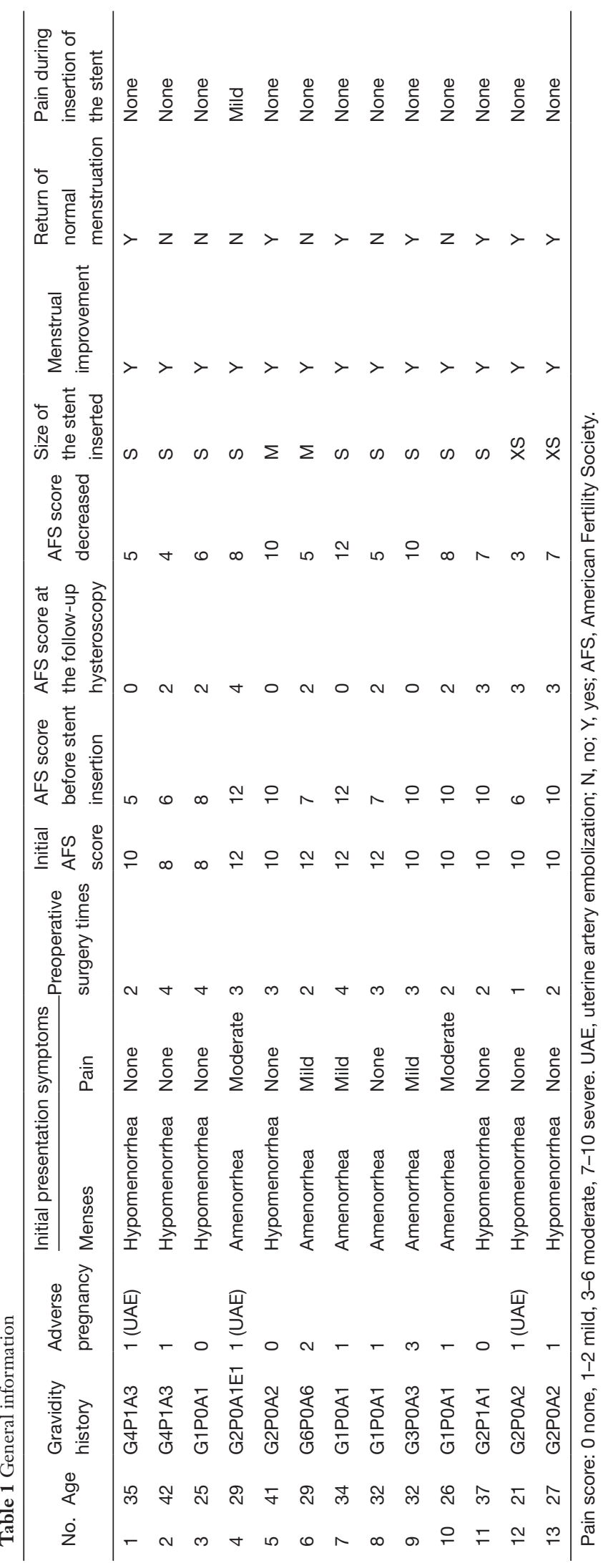

Table 2 Follow-up information

\begin{tabular}{|c|c|c|c|c|c|}
\hline No. & $\begin{array}{l}\text { Follow-up } \\
\text { duration } \\
\text { (month) }\end{array}$ & Recurrence & Menstruation & Pain & Pregnancy \\
\hline 1 & 12 & $\mathrm{Y}$ & Hypomenorrhea & None & $\mathrm{N}$ \\
\hline 2 & 7 & $\mathrm{~N}$ & Hypomenorrhea & None & $\mathrm{N}$ \\
\hline 3 & 4 & $\mathrm{~N}$ & Hypomenorrhea & None & Y \\
\hline 4 & 12 & $\mathrm{Y}$ & Hypomenorrhea & None & $\mathrm{N}$ \\
\hline 5 & 10 & $\mathrm{~N}$ & Hypomenorrhea & None & $\mathrm{N}$ \\
\hline 6 & 12 & $\mathrm{Y}$ & Hypomenorrhea & None & $\mathrm{N}$ \\
\hline 7 & Lost & Lost & Lost & Lost & Lost \\
\hline 8 & 6 & $\mathrm{~N}$ & Normal & None & $\mathrm{N}$ \\
\hline 9 & 13 & $\mathrm{~N}$ & Normal & None & $\mathrm{N}$ \\
\hline 10 & 3 & $\mathrm{~N}$ & Hypomenorrhea & None & $\mathrm{N}$ \\
\hline 11 & 3 & $\mathrm{~N}$ & Hypomenorrhea & None & $\mathrm{N}$ \\
\hline 12 & 3 & $\mathrm{~N}$ & Hypomenorrhea & None & $\mathrm{N}$ \\
\hline 13 & 1 & $\mathrm{~N}$ & Normal & None & $\mathrm{N}$ \\
\hline
\end{tabular}

N, no; Y, yes; NA.

HA, including IUD, Foley balloon catheter, fresh amniotic membranes, and hyaluronic acid gels, recurrence occurs in $14-48 \%$ of patients with IUAs treated with a combination of measures $(4,13,14)$. These patients are also referred to as refractory IUAs or recurrent IUAs. There are also little data to suggest the most efficacious approach for the therapy. The successful treatment effect of IUAs will be greatly reduced without an ideal and effective anti-adhesion barrier. If repeated surgeries are performed but yield little efficacy, gynecologists usually advise these patients to abandon further treatment for fertility.

At present, the patented intrauterine stent, an ideal adhesion prevention barrier is considered capable of maintaining the normal anatomy of the uterine cavity after HA. The stent's design includes, at minimum, the following traits: (I) a uterine shape which can match the uterine cavity well; (II) sufficient effective area to block the anterior and posterior walls of the uterus, which may even extend into the cervical canal to prevent and treat the adhesion of the lower segment of the uterus or cervical canal; (III) a thin film in order to keep the endometrium from ischemia and necrosis; (IV) a variety of sizes designed in order to meet the needs of various sizes of the uterine cavity. Theoretically, as long as the size and position of the intrauterine stent are suitable, no recurrence of adhesions will be seen after the 
insertion of the stent.

All patients included in our study had undergone multiple HA surgeries previously, but there were still large barren area of endometrium and moderate to severe IUAs, which prompted poor prognosis. In general, these patients often require early reintervention a few weeks after HA and repeated procedure. But in our study, it is unnecessary for early assessment with hysteroscopy if only the position of the stent is correct. It is a fact that after the insertion of the stent and 2-3 menstrual cycles, a correct position of the stents and no obvious recurrence of IUAs was observed at the follow-up hysteroscopies. Due to the stent's unique design that can prevent every part of the uterine cavity and cervical canal from recurrence of adhesions, it is reasonable that all patients (6/6) who suffered amenorrhea had restored their menstruation after HA and insertion of the stent. For the same reason, $80 \%$ (4/5) of patients who suffered lower abdominal pain completely disappeared and $20 \%$ $(1 / 5)$ experienced a relief during the placement of the stent. While after removal of the stent, no patient experienced lower abdominal pain, which indicates that the only patient who experienced mild pain with the stent might be explained by the side effect of the stent. The recurrence rate of IUAs and pregnancy outcomes after the removal of the stent is still discouraging. Obviously that fact is owned to our studied population with extremely poor prognosis (most were recurrence severe IUAs with small uterine cavity and extremely poor endometrium). Absolutely, the shrank, scarred uterine cavity and extremely poor endometrium can not be corrected by the stent.

In conclusion, even in the recurrent IUAs patients with very poor prognosis, the patented intrauterine stent has been proved to be very effective in preventing the recurrence of adhesions, on condition that its correct position inside the uterine cavity is achieved. Due to the extremely poor nature of the studied population, the recurrent rate of IUAs and pregnancy rate after the removal of the stent is still discouraging, although the improvement of menstruation is inspiring.

\section{Acknowledgments}

This study was supported by the Hunan Science and Technology Department (Grant No. 2018SK2102) and the Fundamental Research Funds of Central South University (No. 2018zzts265). The authors are grateful for Jeffrey Woo, MD. (Minimally Invasive Gynecological Fellow at Scripps Clinic, San Diego, CA, USA) and Arvind Burjoo,
MAS (Department of Obstetrics and Gynecology, Third Xiangya Hospital of Central South University) for their help with the language editing.

\section{Footnote}

Conflicts of Interest: The authors have no conflicts of interest to declare.

Ethical Statement: The authors are accountable for all aspects of the work in ensuring that questions related to the accuracy or integrity of any part of the work are appropriately investigated and resolved. The study was approved by The Institutional Review Board (IRB) of Third Xiangya Hospital of Central South University. Informed consent was obtained after the procedure was fully explained to all participants and their legal guardians.

\section{References}

1. Rabau E, David A. Intrauterine Adhesions, Etiology, Prevention, and Treatment. Obstet Gynecol 1963;22:626-29.

2. Asherman JG. Traumatic intrauterine adhesions. J Obstet Gynaecol Br Emp 1950;57:892-6.

3. Wallach EE, Schenker JG, Margalioth EJ. Intrauterine adhesions: an updated appraisal. Fertil Steril 1982;37:593-610.

4. Hooker AB, Lemmers M, Thurkow AL, et al. Systematic review and meta-analysis of intrauterine adhesions after miscarriage: prevalence, risk factors and longterm reproductive outcome. Hum Reprod Update 2014;20:262-78.

5. Salma U, Xue M, Sayed ASM, et al. Efficacy of Intrauterine Device in the Treatment of Intrauterine Adhesions. Biomed Res Int 2014;2014:589296.

6. Yu D, Wong YM, Cheong Y. Asherman syndrome-one century later. Fertil Steril 2008;89:759-79.

7. March CM. Management of Asherman's syndrome. Reprod Biomed Online 2011;23:63-76.

8. Shaffer W. Role of Uterine Adhesions in the Cause of Multiple Pregnancy Losses. Clin Obstet Gynecol 1986;29:912-24.

9. The American Fertility Society classifications of adnexal adhesions, distal tubal occlusion, tubal occlusion secondary to tubal ligation, tubal pregnancies, müllerian anomalies and intrauterine adhesions. Fertil Steril 1988;49:944-55.

10. Zhang A, Jamail G, Xue M, et al. Hysteroscopic Intrauterine Adhesiolysis Using the "Ploughing" 
Technique With Cold Scissors. J Minim Invasive Gynecol 2015;22:934-5.

11. Huang H, Cheng C, Johnson G, et al. Hysteroscopic Intrauterine Adhesiolysis Using a Blunt Spreading Dissection Technique with a Double Action Forceps. J Minim Invasive Gynecol 2018;25:583-4.

12. Robinson JK, Colimon LM, Isaacson KB. Postoperative adhesiolysis therapy for intrauterine adhesions (Asherman's syndrome). Fertil Steril 2008;90:409-14.

13. Yang JH, Chen CD, Chen SU, et al. The influence of the location and extent of intrauterine adhesions on recurrence after hysteroscopic adhesiolysis. BJOG 2016;123:618-23.

14. Chen Y, Chang Y, Yao S. Role of angiogenesis in endometrial repair of patients with severe intrauterine adhesion. Int J Clin Exp Pathol 2013;6:1343-50.
Cite this article as: Huang H, Zou L, Zhang A, Zhao X, Xu D, Xue M. A preliminary study on a patented intrauterine stent in the treatment of recurrent intrauterine adhesions with poor prognosis. Ann Transl Med 2020;8(4):57. doi: 10.21037/ atm.2020.01.77 